tionary either for the student or the working mathematician. In its present form it can be of considerable help, but it can hardly be considered authoritative. It is to be hoped that a second edition of increased scope and greater accuracy will be prepared. If the deficiencies of the present edition can be remedied and its good qualities retained, it will be of great value to mathematicians.

R. P. BoAs, JR.

The mathematical theory of plasticity. By R. Hill. Oxford, Clarendon Press, $1950.10+356$ pp. $\$ 7.00$.

Although it is more than eighty years since the foundations of the theory of plasticity were laid by Tresca, Saint Venant, M. Lévy, and others, plasticity is still a very young science. After a first strong wave of interest (about 1913-1930), work in this field has slowly but steadily increased and recent years have seen a marked upsurge of interest; this can best be illustrated by the fact that in 1950 no fewer than four very serious books on the subject appeared, by A. M. Freudenthal (Wiley), by R. Hill, by A. Nadai (McGraw-Hill), and a comprehensive survey report by $\mathrm{P}$. G. Hodge (Brown University Notes); in 1951 followed a textbook by Prager and Hodge (Wiley). Among those works Freudenthal's book differs from the others by its wider scope; plasticity as understood in the other books forms only a chapter, although one of central importance, in Freudenthal's approach, since his viewpoint is primarily that of a physicist and technologist. Hill's book is an advanced and comprehensive text, intended as an orientation for engineering scientists and applied mathematicians rather than as a textbook for students; on the other hand Hodge and Prager's useful and interesting book is planned for students on an intermediate level.

Hill's important book presents those aspects of plasticity which so far have been more or less "mathematicised"; by this word we mean that a rational theory which forms part of a larger scientific unit (the science of mechanics) is formulated in mathematical terms. This is true in particular for that part of plasticity theory which is known today as the theory of the "ideal" or "perfect" plastic body. Such a body is described mathematically by the system of equations at the basis of the theory. We may, however, point out a few features: (a) The equations deal only with stresses and deformations at a fixed moment; after the whole configuration is determined for an instant the investigation may be repeated if necessary for the next moment; (b) thermal phenomena are disregarded; (c) work hardening and related phenomena are in general neglected, etc. 
The characteristic features of an ideal plastic material, as contrasted to an elastic one and described in the equations of plasticity, are very roughly the following: (1) While in an elastic material there is a one to one correspondence between strain and stress, a completely plastic material flows under constant stress (if only one stress component $\sigma$ matters, then, $\sigma=\sigma^{*}$; or, in general, a certain function of the stress tensor remains constant throughout the plastic flow). (2) A relation is assumed between stress tensor and (total) strain velocity tensor (rather than between stress tensor and (elastic) strain tensor). Mathematically, the whole setup consists of ten equations (nine differential equations and one finite equation) for the following ten unknowns: six components of the symmetric stress tensor, $\Sigma$, three components of the flow velocity vector, $\bar{v}$, and a nonnegative scalar function $\lambda(x, y, z)$. The equations are (a) three equilibrium equations for $\Sigma$, (b) the yield condition, a finite equation which singles out the five-dimensional manifold of admissible stresses, (c) the six relations between stress and strain velocity; in the fully plastic state stresses are proportional to strain rates with the factor of proportionality $\lambda$. This looks similar to the basic relation of viscous fluid theory; the decisive difference is, however, that there the proportionality factor $\mu$, the viscosity, is a material constant while our $\lambda$ is an unknown function of the space coordinates. Of these last six equations five only are independent since they are such that incompressibility holds, in the form $\operatorname{div} \bar{v}=0$; these equations, generally called the Lévy-Mises or Saint Venant-Mises equations, are strictly applicable only to a fictitious material in which elastic strains are zero. The strain equations of Prandtl (1924) and Reuss (1930) take into account not only plastic but also elastic strains. At any rate the complete general system of ten equations is too complicated for actual applications.

The first three chapters of Hill's work form a preliminary unit, where the physical and mathematical foundations are explained (for the general three-dimensional case) and some general theorems, statements of uniqueness, some extremum principles, etc., are considered. A second group, in Chapters IV and V, concerns some plastic-elastic problems. As long as the loads acting on a metal are sufficiently small, the body is elastic; as the loads are increased plastic regions begin to appear which successively grow in size; the plastic zones are, however, restrained from actual flowing ("contained plastic state") by the remaining still elastic material (think of the expansion of a cavity by internal pressure in a thick-walled shell). Finally the plastic regions may spread and merge to such an extent as to make actual 
plastic flow possible; then the elastic deformation is negligible compared to the much larger plastic deformation, and the corresponding idealization is a rigid plastic type of material with the Lévy-Mises equations valid. In problems of contained plastic strain the material is plastic-elastic; the complete solution, based on the Prandtl-Reuss equations, involves a calculation of stress and deformation in the elastic as well as in the plastic region; the boundary between these regions is in general unknown (the same is true for the rigid plastic boundary in the other problems). So far only elastic-plastic problems of a simple type have been solved; it may, e.g., happen that all elements of the metal reach the yield limit simultaneously so that no plastic-elastic boundary need be found, as in the combined torsion and tension of a cylindrical bar; in other problems, like that of the torsion of a prismatic bar, elastic and plastic zones exist side by side but other simplifications prevail. Then follow the problems, extensively investigated by several authors, of the expansion of a spherical shell and of a cylindrical tube by internal pressure; the presentation is based on the author's own work (with coauthors).

The central part of the book, formed by Chapters VI-IX, deals, for plane motion, under conditions of "plane strain," with problems where a plastic rigid material may be assumed. Here we have a type of problem amenable to mathematical methods. For the fully plastic material there are now six equations and unknowns (or five after elimination of $\lambda$ ); the unknowns are the three components $\sigma_{x}, \sigma_{y}$, $\tau$ of $\Sigma$, the two components $v_{x}, v_{y}$ of $\bar{v}$ and $\lambda$; the equations are (a) two equilibrium conditions, (b) the yield condition in the form $\left(\sigma_{x}-\sigma_{y}\right)^{2}+4 \tau^{2}=$ const., (c) three relations between the stress and strain rate tensors. Here, (a) and (b) are three equations for $\sigma_{x}, \sigma_{y}, \tau$ alone, so that the five equations fall into two sets, as long as no boundary conditions are considered. The set (a), (b) is nonlinear but of the "reducible" type, i.e., after elimination of one stress-variable by means of (b) there remain two partial differential equations which, as in compressible fluid theory, can be transformed into two linear equations, for example by means of an exchange of dependent and independent variables. The theory of these stress equations has been extensively investigated. If the tensions are known, the last two equations for the velocities are linear; it turns out that the characteristics of the "stress equations," and of the "velocity equations" are always real, coincide with each other, and bisect the angles of the principal stress directions (which are also the principal strain rate directions). The elegant theory of this "slip line field" is clearly and completely exposed in VI, including also some numerical methods based on the 
finite difference equivalents of the basic equations referred to the slip lines.

Chapters VII-IX deal with applications of the theory to actual problems. Unfortunately the "static determinacy" of the stress equations is in general lost in actual problems, since the boundary conditions concern stresses and velocities. While one can always find partial solutions of the stress equations, without considering velocities, the correct stress solution of a specific problem can be found in this way only if there are sufficiently many pure stress-boundary-conditions. In this case, after the correct stresses have been found, we may solve the boundary problem of the second group, i.e., of the velocity equations. If, however, the boundary conditions do not fall into two such groups, the whole problem must be dealt with more or less simultaneously. In addition, the boundaries between rigid and plastic regions are not known beforehand so that we have certain conditions along free boundaries. Hence the solution will often be based on a method of systematic trial and error where we experiment with assumed plastic rigid boundaries, or with guessed solutions. In addition to these essentially mathematical difficulties, a great difficulty in boundary problems of plasticity consists often in the definition and setting up of the problem, that is, in the determination of the right boundary conditions. In this difficult task one would much like to complement the mechanical knowledge and intuition by means of mathematical information, which, however, is of ten not available; we are thinking of general existence theorems as they exist in hydrodynamics or elasticity theory, which would help to decide what groups of stress- and velocity-boundary conditions determine a solution.

However, a considerable number of actual problems have been set up, solved, and discussed, particularly in recent years, and Hill has contributed much to this work. Chapter VII deals with so-called "steady state" problems, i.e., problems where stress- and velocityfield do not vary in time. The history of the changes through which the steady plastic state is reached is not taken into account. The chapter contains much of the author's own work. It would lead too far to talk to mathematical readers about "sheet drawing," "sheet extrusion," etc. Non-steady problems are those where stress-and velocity-field are varying in time; this variation may be such as to preserve geometric similarity ("quasisteady"), or no such restriction may hold. Again much of the author's work is incorporated; problems of wedge indentation, of compression of a wedge, etc., belong to this class. If, however, a rectangular block of plastic rigid material is 
compressed between rough rigid plates (shorter than the block), geometric similarity is not maintained. Finally, in Chapter IX, plane problems are discussed which require the consideration of an elasticplastic material.

In Chapter XI, which deals with "miscellaneous topics," two further mathematically similar plane cases are discussed, "plane strain of a more general plastic material," and "plane stress." Both make it necessary to abandon the particular simple yield condition mentioned above, a fact that greatly complicates the mathematical situation. While the basic equations in VI-IX are everywhere hyperbolic, with real orthogonal characteristics, the more general equations are in general partly hyperbolic, partly elliptic or parabolic (and no longer orthogonal); since the stress equations are not linear, the regions of this varying behaviour depend on the specific solution considered. Essential parts of the theory are given; some of the results seem to be true under more general conditions than those stated.

Chapter $\mathrm{X}$ gives the basic equations and some applications for the case of axial symmetry. Here, just as in the plane problem, there are only two independent variables; there are, however, four nonvanishing stress components, and the stress equations, as they are, are not statically determined. Chapter XII offers an introduction to nonisotropic problems.

All through the book there are very complete bibliographical notes, in addition to an author index and a subject index at the end of the book.

The reviewer admires Hill's accomplishment: in addition to reporting so much published work, he has filled out many gaps by original remarks and contributions, thus achieving a unified presentation. It may, however, be said that, occasionally, when the reviewer sought instruction regarding a specific problem it was found very hard to follow the explanations. Returning, in such a case, to the original paper-by Hill-the desired information was readily obtained. Probably such things are unavoidable in a work so rich in content. By the way, the reviewer wonders why the useful tool of graphing a streamline pattern in order to impart in one view a picture of a velocity distribution is not used (in problems of plane strain a simple stream function, $\psi(x, y)$, exists with $\left.\partial \psi / \partial x=v_{y}, \partial \psi / \partial y=-v_{x}\right)$. There were instances when the reviewer could not approve of a mathematical presentation or of an argument. But at any rate, even apart from the question of taste, such details are of minor interest, if, as in this book, the difficult task of expounding critically and in a unified way so much novel research work has been so successfully ac- 
complished. We join the author in his hope that his work "will attract engineers and applied mathematicians to a field which well rewards study and research."

\section{H. GeIRINGER}

Fourier transforms. By I. Sneddon. New York, McGraw-Hill, 1951. $12+542$ pp. $\$ 10.00$.

It is the aim of the author to discuss various types of integral transforms from an elementary mathematical viewpoint and to demonstrate how they may be applied to various boundary value problems which arise in the physical and engineering sciences. Accordingly, some basic aspects of these transforms are discussed in the first three chapters of this text. Chapter one is concerned with the Fourier, Laplace, and Mellin transforms for one variable as well as the multiple Laplace and Fourier transforms. It is unfortunate that the complex form of the Fourier transform was not included here, for then one could see that there is no basic distinction between these transforms. That is, what may then be accomplished by the unilateral transform of Fourier may be equally well accomplished by the unilateral Laplace transform, etc. The second chapter contains a discussion of Hankel transforms (real case) as well as the relation between the real multiple Fourier transform and Hankel transforms. It closes with a discussion of dual integral equations of a special class which has been discussed by Titchmarsh and his collaborators. The closing chapter of this part of the book is devoted to a discussion of the finite Fourier and Hankel transforms. These transforms are infinite series of the Fourier or Fourier-Bessel type which arise naturally in SturmLiouville expansion theory. The application of these finite transformations to appropriate boundary value problems simply states that one is aware of the correct form of the expansion in advance.

The remaining seven chapters are concerned with the applications of these mathematical methods to many ordinary and partial differential equations which arise in the physical and engineering sciences. No specialized knowledge of physics is assumed and the remaining background is discussed with the view of supplying the necessary differential equations and their subsidiary conditions. We find, in the second portion of the book, applications drawn from vibration theory, elasticity, hydrodynamics, and heat conduction as well as some problems drawn from atomic and nuclear physics.

The book closes with three appendices. The first one is concerned with some properties of Bessel functions, while the second one discusses the method of steepest descent and some numerical methods. 